\title{
The wireless data acquisition system based on Android system
}

\author{
Huaqun Zhan ${ }^{\mathrm{a}}$, Huarong Chen ${ }^{\mathrm{b}}$ and Wenjie $\mathrm{Wu}^{\mathrm{c}}$
}

Jiangxi Science and Technology Normal University, School of communication and electronic,

Nanchang, 330013, China

a4492319165@qq.com, b313473168@qq.com, '583408852@qq.com

\begin{abstract}
Keywords: Information processing, Acquisition system, Android, Bluetooth, Data transmission.
\end{abstract}
\begin{abstract}
Aimed at the defects of data acquisition systems such as poor versatility, poor mobility, time and effort wasted, poor flexibility, vulnerable to adverse environmental, a wireless data acquisition system based on Android system was proposed. The system took the chip named STC12C5616AD as the main controller which can read and process data from sensor, took the universal Bluetooth serial port module HC-06 as data wireless transmission medium, a Android smart phone displays data and curve real-time. This system realized the wireless acquisition of data and the show of data real-time. The experimental results indicate that the data acquisition system designed by the program meets the requirements for commonality, real-time, low-cost, mobility, stability and reliability. It has a broad application prospect in the fields of electronic experiment, industrial monitoring, and medical care, etc.
\end{abstract}

\section{Introduction}

As one of the preliminary work of signal processing, data acquisition is widely used in military, industrial, communications, medical, and other measurement and control fields. Acquisition and collection of data are expected to be low cost, high speed transmission, not restricted by environment, wireless transmission and easy to carry. Bluetooth technology provides low cost, close range of wireless communication, constitute a personal network in communication environment between fixed and mobile devices, enables the close range of all sorts of equipment to achieve seamless resource sharing, replace cable connection with wireless connection, allowing mobile users to get rid of the shackles of the cable, realize low-cost wireless interconnection communication between devices. In order to achieve the purpose of data collection more convenient and lower cost, this paper designed a data acquisition system based on Android system and Bluetooth module to display data real-time on Android smart phone that are collected by the acquisition module and sent by bluetooth module. Different data can be easily collected by changing data acquisition sensor.

\section{The hardware circuit design}

\subsection{General structure of hardware system}

System consists of two parts, data sending module and data receiving module, wireless data transmit between the sending module and the receiving module through bluetooth module. The main function of the data sending module is to receive and process the data collected by the sensor, and then send the data out by the Bluetooth module. With the phone's built-in bluetooth module, Android phone establishes a connection with the bluetooth signal of the data sending module by searching bluetooth devices, receives and processes and display curve of the sensor data real-time. Block diagram of the system is shown in Fig. 1.

\subsection{Hardware circuit devices}

The hardware circuit of the system uses STC12C5616AD micro-controller as the main controller, and uses the universal bluetooth serial port module HC-06 as data transmission medium.

HC-06 bluetooth module provides a complete bluetooth solution, using CSRBC04 bluetooth chip technology, built-in $2.4 \mathrm{GHz}$ antennas, 8Mbit external Flash, self-adaptive frequency hopping 
technology, Class2 power level, equipped with Bluetooth 2.0 with EDR, the highest chip transmission rate can reach 3Mbps, is a widely applied standard. The Host Controller Interface (HCI) transport layer can be achieved through the UART/USB. Communicate with a computer or any microprocessor through wireless UART (Universal Asynchronous Receiver/Transmitter) serial port. HCI transmits data, events and commands in the form of grouping.
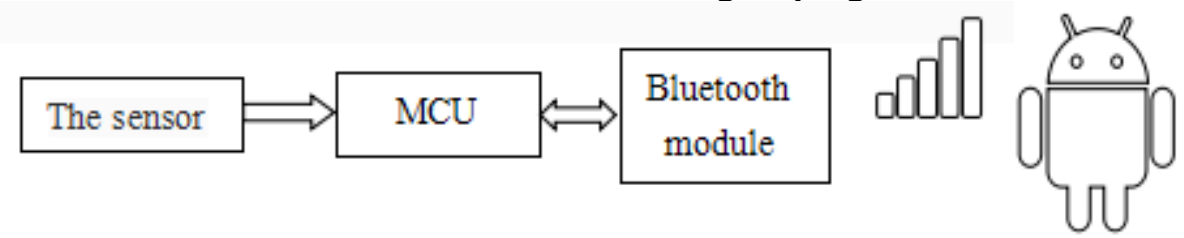

The data sender

Data receiver

\subsection{The sender hardware design}

Fig. 1 Overall system block diagram

Sending module has three parts: sensor, micro-controller STC12C5616AD, bluetooth module HC-06. The MCU and bluetooth module are core components, build up intercommunication between the two via serial port operation instructions. MCU send the sensor data that have been processed to the bluetooth module. The sender hardware connection diagram is shown in Fig. 2.

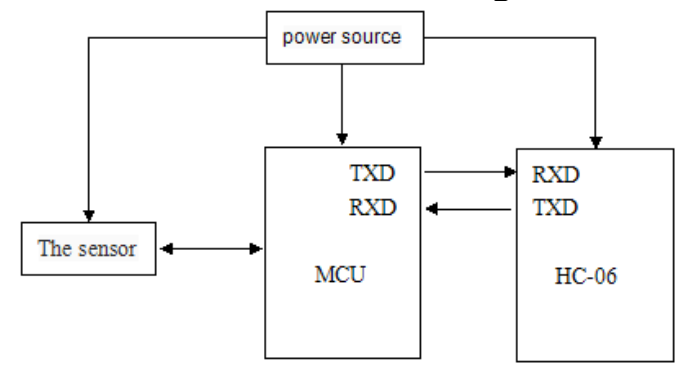

Fig. 2 The sender hardware connection diagram

\section{Software design}

\subsection{MCU program design}

Bluetooth module HC-06 being used is a serial bluetooth module. Parameters can be configured through the serial port AT command mode. During the single chip microcomputer programming, also needs to configure the serial port work mode of the single chip microcomputer. The micro-controller reads the sensor data and process them, the data would be sent to the bluetooth module via a serial port after that. Wireless data can be transmitted between data sending module and cell phone after the connection between bluetooth modules of sending module and cell phone being established. This system set the serial port work mode to the mode 1,8 bits of data, and permitted to accept, baud rate to $9600 \mathrm{Bd}$.

\subsection{The receiver program design}

The receiver uses the Android smartphones. Android is Google's open source mobile operating system based on Linux platform, using software stack layer architecture, mainly divided into four layers: Bottom layer(Linux Kernel), System runtime layer(Library), Application framework layer (Application Frame) and the Application layer (Application). This paper enables the development of data transmission system at the Application layer. Android version 2.0 begin to support bluetooth. The classes and interfaces about bluetooth are in the android. Bluetooth package.

\subsection{The realization of bluetooth basic operation}

In order to realize the function of bluetooth in the Android system, there are some preparations to be done. First of all, set up to obtain the corresponding access instructions in the file AndroidManifest.xml, as follows:

<Uses-permission android: name="android. Permission. BLUETOOTH_ADMIN"/>

$<$ Uses-permission android: name="android. Permission. BLUETOOTH”/> 
Second, to obtain a bluetooth adapter. The local bluetooth adapter can be obtained by get Default Adapter (), get remote bluetooth adapter by using the Bluetooth Device class. After getting the bluetooth adapter, request to open the bluetooth devices and began to search bluetooth devices around.

To search remote bluetooth devices, use Bluetooth Adapter. Get Remote Device () to get the Bluetooth Device with specified address. The class is actually a remote Bluetooth hardware address creates a Bluetooth Device object with Bluetooth Adapter. After the program starts, register two Broadcast Receiver, ACTION_FOUND and ACTION_DISCOVERY_FINISHED, then control the search for bluetooth devices (start Discovery) through the thread. When the search is triggered by the two receiver events above, it would be transmitted to the receiver to save. Program flow chart is shown in Fig. 3.

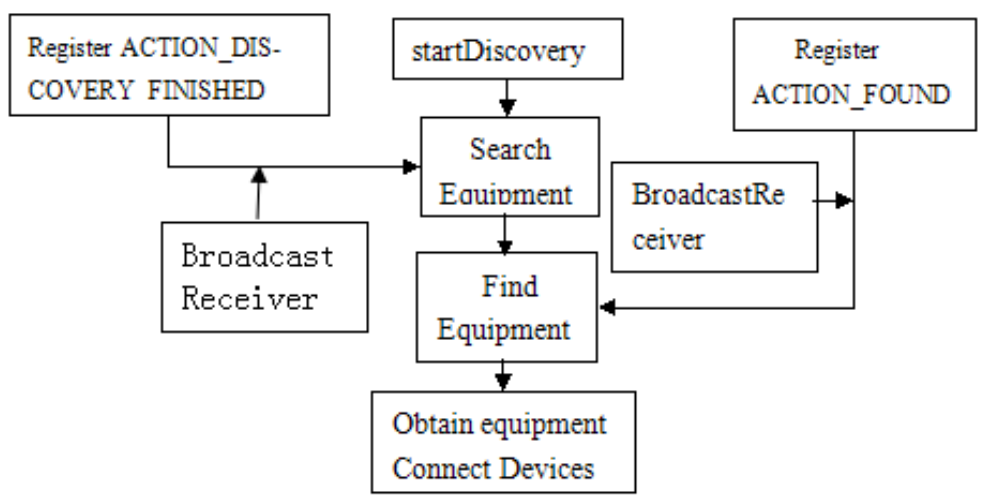

Fig. 3 Search equipment flow chart

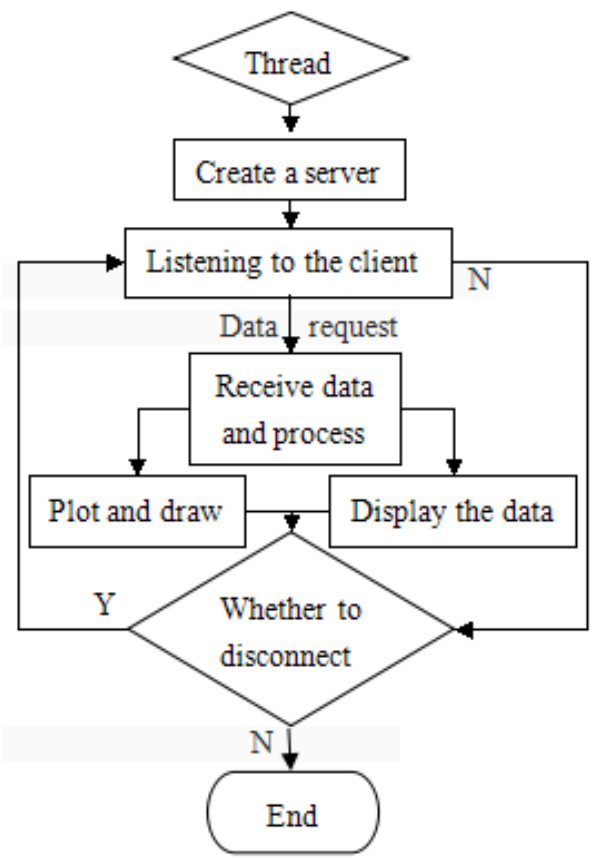

Fig. 4 The flow chart of the server

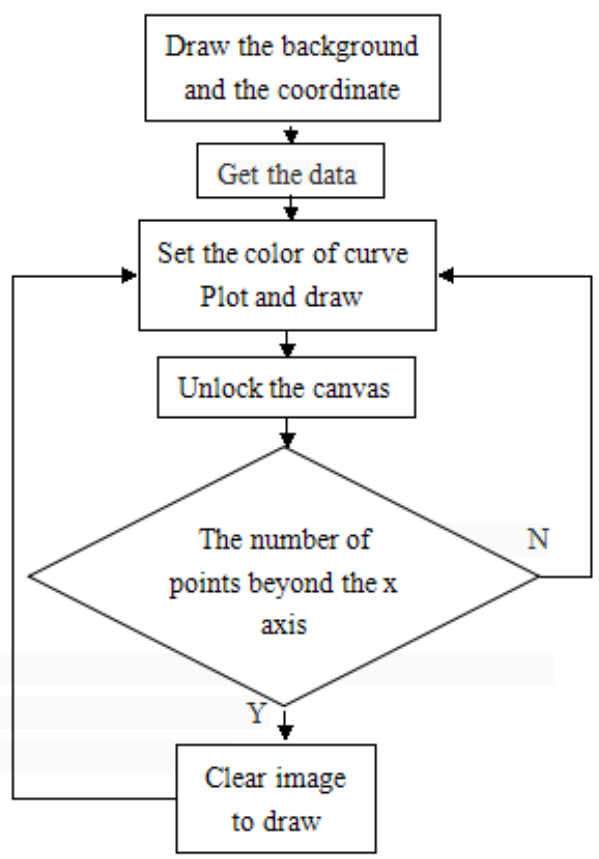

Fig. 5 Drawing flow chart

\subsection{The data receiving and display}

In this system, the phone server uses listen Using Rfcomm With Service Record() method to register Bluetooth Server Socket with a name and an unique identification UUID number, then use Bluetooth Server Socket. Accept () method to keep listening to the request of the data acquisition module. After getting request, use Input Stream. Read (byte [ ]) method to take the data and process the data. When need to close the Bluetooth server, call Bluetooth Server Socket. Close () method. Program flow chart is shown in Fig. 4.

Real time curve of data is drawn by using Canvas () method of Surface Holder in Android Surface View. Plot the curve with draw Point () method of Canvas class. The draw Rect () method can be used to determine the drawing area. The set Color () method of Paint class can set the color of the curve. Program flow chart is shown in Fig. 5. 


\section{The results show}

Mobile terminal APP interface is shown in Fig. 6. Search equipment results is shown in Fig. 7. In this paper, there are two tests, the first test is transmission and display of Sine voltage sampled data, in this test, the sensor part of system is Sine voltage sampling circuit, test result is shown in Fig. 8. The second test use temperature sensor DS18B20 as the sensor part, touch the temperature sensor with fingers during test, after a period of time, release fingers, the temperature curve is shown in Fig. 9.

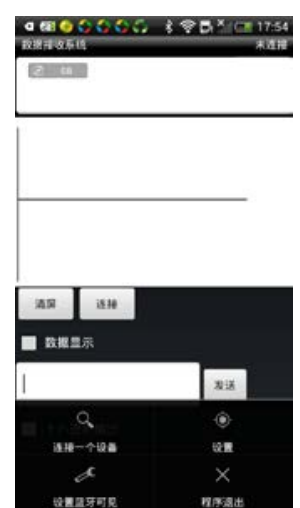

Fig. 6 APP interface

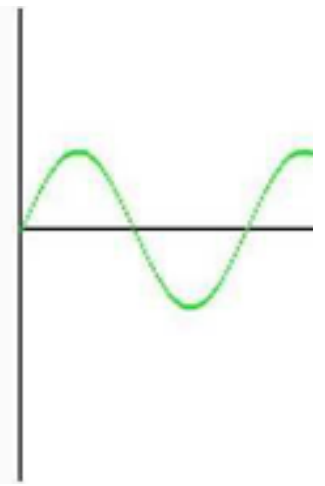

Fig. 8 Sine voltage curve

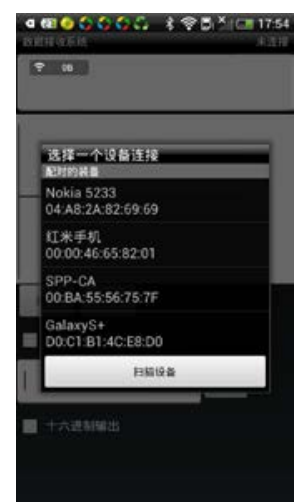

Fig. 7 Search equipment

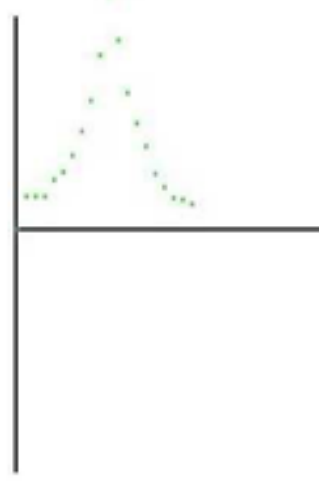

Fig. 9 Temperature curve

\section{Conclusion}

The wireless data acquisition system proposed in this paper based on Android system, combined with the wireless transmission function of Bluetooth module, achieves acquisition and processing of data. The advantages of Bluetooth technology such as low cost, low power consumption, small volume and the popularization of Android provide this system a broad stage and significant application value in the fields of electronic experiment, industrial monitoring, and medical care,etc.

\section{References}

[1]. Luan Lvhai, Wang Meng. Research and Implementation of Bluetooth-based multi-channel wireless test system [J]. Microcomputer Information, 2007, 23 (11): 265-266.

[2]. Zhou Hualan, Tang Minghao. The application of bluetooth technology in wireless distributed measurement and control system [J]. Journal of electronic technology, 2003 (4): 28-30.

[3]. Zhang Qun, Yang Xu, Zhang Zhengyan, etc. The design and implementation of serial communication with bluetooth module [J]. Journal of laboratory research and exploration, 2012, 31 (3): 79-82.

[4]. Yang Fengsheng. The Android application development revelation [M]. Beijing. Mechanical industry press. 2011:5-7.

[5]. Zhang Yigang. The principle and application of single chip microcomputer [M] Beijing. Higher education press. 2004. 